\title{
LANGUAGE AND SPEECII PROBLEMS RELATED TO CARBAMAZEPINE IN BENIGN ROLANDIC EPILEPSY
}

Language and speech problems in benign rolandic epilepsy (BRE) and changes occurring after treatment with carbamazepine (CBZ) monotherapy were investigated in 11 patients, ages 5 to 12 years, treated at Chonbuk National University, Medical School, Jeonbuk, Republic of Korea. All patients had multiple seizures before treatment. Laryngeal articulation errors in stop consonants found in children with BRE increased from $6 \%$ before CBZ treatment to $13 \%$ after treatment. A muscle weakness in phonations, suggested by a decrease in habitual pitch and vowel formants (F1 and F2), is attributed to CBZ-induced decrease in nerve conduction velocity. Acoustic abnormalities caused by CBZ during treatment of BRE resemble the hypokinetic pattern of articulation abnormalities. (Park J-I, Kim SJ, Kim H-G. Acoustic effects of carbamazepine in benign rolandic epilepsy. Epilepsy Behav November 2005;7:468-471). (Respond: Jeong-In Park MD, Department of Pediatrics, Chonbuk National University, Medical School, Jeonju, Jeonbuk, Republic of Korea).

COMMENT. The treatment of BRE with carbamazepine is controversial, most authorities reserving treatment for patients having frequent seizure recurrences with secondary generalization. BRE seizure patterns involve motor, sensory, and autonomic manifestations in face, mouth, and tongue, and include drooling, guttural sounds, involuntary movements of tongue or jaw, unilateral numbness of tongue, lips, gums, and cheek, speech arrest, and myoclonic jerks of one side of the face. Speech and language impairments may be expected since epileptiform discharges involve centrotemporal speech centers. CBZ is known to affect motor conduction and neuromuscular transmission, adding to the likelihood of phonation and articulation errors. Previous reports have alluded to oral and speech dyspraxia in children with BECTS (Roulet E et al. 1989; Scheffer IE et al. 1995; Ped Neur Briefs Oct 2005;11:76-77). BRE is no longer regarded as entirely "benign." Patients treated with CBZ should be monitored for language and speech problems. If the adverse effects of AEDs on cognitive function and speech could be avoided, and interictal epileptiform discharges suppressed, the prognosis for BRE would be more favorable. The following report advocating treatment of BRE holds some promise.

Oxcarbazepine (OXC) monotherapy in BECTS. A trial of OXC in 70 patients (aged 5-11 years) with benign childhood epilepsy with centrotemporal spikes (BECTS) demonstrated effective prevention of seizures, normalization of EEG, and preservation of cognitive and behavioral functions (Tzitiridou M et al. Epilepsy Behav Nov 2005;7:458-467). None developed regression of language function. The authors recommend treatment of BECTS with OXC, citing the lack of cognitive and behavioral deficits associated with some AEDs, and accelerating the time to spontaneous recovery.

Oxcarbazepine adjunctive therapy in infants and young children with partial seizures was found more effective in high doses $(60 \mathrm{mg} / \mathrm{kg} /$ day $)$ than low doses $(10 \mathrm{mg} / \mathrm{kg} /$ day $)$, in a multicenter, rater-blind, randomized study involving 128 patients $(1$ month to $<4$ years of age). Somnolence, pyrexia, ataxia, and vomiting were the most frequent adverse events. OXC is approved for treatment of adults and children $>4$ years in US. (Pina-Garza JE, Espinoza R, Nordli D et al. Neurology Nov (1 of 2) 2005;65:1370-1375). 